\title{
Genetically Encoding Photocaged Quinone Methide to Multi- Target Protein Residues Covalently in vivo
}

\author{
Jun Liu ${ }^{1, \ddagger}$, Shanshan Li ${ }^{1,2, \ddagger}$, Nayyar A. Aslam ${ }^{3, \ddagger}$, Feng Zheng ${ }^{3}$, Bing Yang ${ }^{1}$, Rujin Cheng ${ }^{4}$, \\ Nanxi Wang ${ }^{1}$, Sharon Rozovsky ${ }^{4}$, Peng G. Wang ${ }^{2}$, Qian Wang ${ }^{3}$, Lei Wang ${ }^{1,}$, \\ ${ }^{1}$ Department of Pharmaceutical Chemistry and the Cardiovascular Research Institute, University \\ of California San Francisco, 555 Mission Bay Boulevard South, San Francisco, California 94158, \\ United States \\ 2Department of Chemistry and Center for Therapeutics and Diagnostics, Georgia State University, \\ Atlanta, Georgia 30302, United States \\ ${ }^{3}$ Hangzhou Research Institute of Technical Institute of Physics and Chemistry, Chinese Academy \\ of Sciences, Hangzhou 310018, China \\ ${ }^{4}$ Department of Chemistry and Biochemistry, University of Delaware, Newark, Delaware 19716, \\ United States
}

\begin{abstract}
Genetically introducing covalent bonds into proteins in vivo with residue specificity is affording innovative ways for protein research and engineering, yet latent bioreactive unnatural amino acids (Uaas) genetically encoded to date react with one to few natural residues only, limiting the variety of proteins and the scope of applications amenable to this technology. Here we report the genetic encoding of (2R)-2-amino-3-fluoro-3-(4-((2-nitrobenzyl)oxy) phenyl) propanoic acid (FnbY) in $E$. coli and mammalian cells. Upon photoactivation, FnbY generated a reactive quinone methide (QM), which selectively reacted with nine natural amino acid residues placed in proximity in proteins directly in live cells. In addition to Cys, Lys, His and Tyr, photoactivated FnbY also reacted with Trp, Met, Arg, Asn and Gln, which are inaccessible with existing latent bioreactive Uaas. FnbY thus dramatically expanded the number of residues for covalent targeting in vivo. QM has longer half-life than the intermediates of conventional photo-crosslinking Uaas, and FnbY exhibited crosslinking efficiency higher than $p$-azido-phenylalanine. The photoactivatable and multi-targeting reactivity of FnbY with selectivity toward nucleophilic residues will be valuable for addressing diverse proteins and broadening the scope of applications through exploiting covalent bonding in vivo for chemical biology, biotherapeutics, and protein engineering.
\end{abstract}

\section{Graphical Abstract}

\footnotetext{
*Corresponding Author: Lei.wang2@ucsf.edu.

¥These authors contributed equally.

Supporting Information. The Supporting Information is available free of charge on the ACS Publications website.

Experimental details and Figures S1-S6 (PDF)
} 


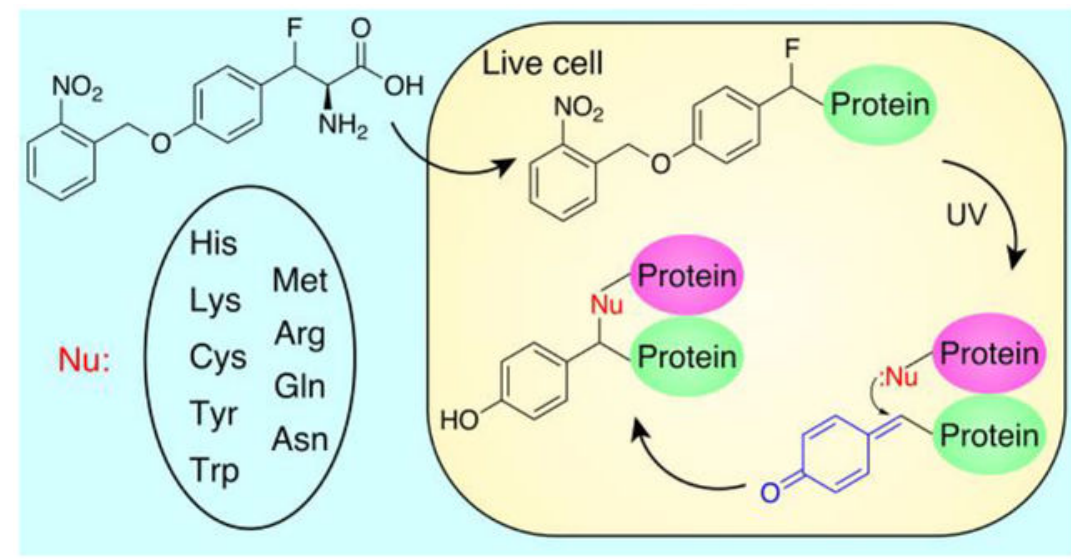

The ability to selectively introduce covalent linkages among amino acid side chains of proteins in vivo would afford new avenues for protein research, protein engineering, biotherapeutics, and synthetic biology. ${ }^{1,2}$ Genetically encoded photocrosslinkers react with all natural amino acid residues but in a nonselective manner. Although valuable for crosslinking of proteins with interacting partners, ${ }^{3-6}$ such nonselective reactivity makes it difficult to identify the crosslinked residues using mass spectrometry, and has rarely been utilized for judicious engineering of covalent bonds into proteins. Recently, selective covalent bonding of proteins has been enabled by the genetically encoding of latent bioreactive unnatural amino acids (Uaas). ${ }^{1,2}$ These Uaas have fine-tuned reactivity compatible for use in cells. After being incorporated into proteins, they react with nearby target natural residues specifically via proximity-enabled reactivity, forming covalent linkages selectively in living systems. The new covalent bonding ability has been harnessed within proteins to enhance or create protein properties, ${ }^{1,7-10}$ and between proteins to study protein interactions and to generate irreversible binders and inhibitors. ${ }^{1-16}$ Latent bioreactive Uaas have been developed to react with Cys, Lys, His, Glu, and Tyr. 1,7,10,12,13,17-19 However, other natural residues have not been targeted successfully, and each latent bioreactive Uaa can usually react with one or up to three natural residues only.

The ability of a bioreactive Uaa to react with a broader range of natural residues will expand the scope of this methodology and provide flexibility for applications. For instance, when identifying unknown protein-protein interactions wherein no information of the target natural residue is available, a bioreactive Uaa with broader reactivity will significantly increase the chance of covalent capturing. Here we report the genetically encoding of a new latent bioreactive Uaa FnbY in E. coli and mammalian cells, which upon photoactivation is able to react with nine different natural residues via proximity-enabled reactivity in vivo.

Genetically encoding a bioreactive Uaa with multi-targeting ability is challenging because high reactivity is needed yet it tends to cause cytotoxicity and lose reaction specificity. We reasoned that this challenge can be overcome by combining the advantage of photocrosslinkers (i.e., high reactivity masked by photocage) with that of proximity-enabled reactivity (i.e., specificity achieved by proximity). We decided to genetically encode a photocaged reactive intermediate that has reaction selectivity, for which quinone methide 
(QM) was chosen. QM is a highly reactive Michael acceptor for nucleophiles. It occurs as natural metabolic intermediates of biosynthesis and has been widely explored for organic synthesis and chemical biology, ${ }^{20-27}$ but has not been genetically incorporated into proteins.

To genetically incorporate a photocaged QM in live cells, we designed a Uaa $(2 R)-2-$ amino-3-fluoro-3-(4-((2-nitrobenzyl)oxy)phenyl)propanoic acid ( $\alpha$-2-nitrobenzyl- $\beta$-Ftyrosine, FnbY) by installing a fluorine at the $\beta$ position of $\alpha$-2-nitrobenzyl-L-tyrosine (Figure 1a). When the photocaging $o$-nitrobenzyl group is released by UV light, the fluoride ion will be eliminated to generate QM spontaneously. The QM generated in situ will then allow for Michael addition of a nearby nucleophilic natural residue, forming a covalent bond selectively. To evolve an aminoacyl-tRNA synthetase specific for FnbY, we constructed a focused mutant library of Methanosarcina barkeri pyrrolysyl-tRNA synthetase (PylRS) and subjected it for selection. Multiple hits showing FnbY-dependent phenotype were identified and converged on the same amino acid sequence (L270F/L274M/N311A/C313G) (Supplementary methods), which we named as FnbYRS. To verify FnbY incorporation, we expressed in E. coli the glutathione transferase (GST) gene containing a TAG codon at position 103 together with $\mathrm{tRNA}_{\mathrm{CUA}}^{\mathrm{Py}} / \mathrm{FnbYRS}$. In the absence of FnbY, no full-length GST was detected; when $1 \mathrm{mM}$ of FnbY was added in growth media, full-length GST was expressed and purified (Figure 1b). The purified GST(103FnbY) was analyzed with electrospray ionization time-of-flight mass spectrometry (ESI-TOF MS), which confirmed the successful incorporation of FnbY (Figure 1c).

We next determined whether the incorporated FnbY could react with lysine in proximity upon photoactivation directly in E. coli cells. GST is a dimeric protein, whose structure shows that residue 103 of one monomer is close to residue 107 of the other monomer at the dimer interface (Figure 2a). ${ }^{28}$ We thus incorporated FnbY at site 103 and tested if it could covalently crosslink with the native Lys 107 upon photoactivation (Figure 2b). E. coli cells expressing GST(103FnbY) were illuminated with UV light, and cell lysates were analyzed with Western blot to detect GST. GST dimerization was detected upon UV light activation (302 $\mathrm{nm}$ or $365 \mathrm{~nm}$ ) for $5 \mathrm{~min}$, with efficiency reaching 75\% (Figure 2c, Figure S1). When using a wild type GST (no FnbY) or mutating Lys107 to Ala (103FnbY/107Ala), no GST dimeric crosslinking was detected under identical conditions (Figure S2). These results suggest that the covalent crosslinking occurred between FnbY103 and Lys107 selectively. We further used tandem mass spectrometry to determine the bonding pattern of the crosslinked GST. The cross-linked GST was purified, digested with endoproteinase GluC, and analyzed with nano-LC-MS. The fragmented ions in MS2 spectrum indicate that the covalent linkage formed between Lys107 of one GST monomer with Tyr103 of the other monomer (Figure 2d). Because QM generated from FnbY would be converted into a Tyr upon Michael addition (Figure 2b), the MS result thus further confirms that the reaction occurred between FnbY103 and Lys107 via the QM mechanism.

To test if FnbY could react with other natural residues, we mutated Lys107 of GST to all other possible nucleophilic amino acids, including His, Tyr, Cys, Trp, Ser, Thr, Asp, Asn, Glu, Gln, Met, and Arg. E. coli cells expressing GST containing FnbY103 and each test residue at site 107 were photoactivated and analyzed with Western blot (Figure 3). To our 
delight, the FnbY-generated QM reacted with His, Tyr, Cys, and Trp in high efficiencies up to $80 \%$. Remarkably, it also reacted with Met, Arg, Asn, and Gln, which have weak nucleophilicity, in moderate efficiencies (9\% - 29\%). For Ser, Thr, Asp, and Glu, GST dimerization could be detected but the reactivity was low. The crosslinking of FnbY103 with His107, Tyr107, and Cys107 were further verified with tandem MS (Figure S3).

QM photo-released from FnbY showed broad reactivity toward natural amino acids, yet it is distinct from conventional photo-crosslinking Uaas containing phenyl azide, diazirine, or benzophenone. First, in terms of reaction mechanism, FnbY generates an electrophilic Michael acceptor QM to react with nucleophilic Michael donor selectively, while photocrosslinking Uaas generate nitrene, carbene, or diradical, which react with any natural residue non-selectively. Second, the intermediates generated from photo-crosslinking Uaas have very short half-life (ns to $\mu$ s), ${ }^{29,30}$ whereas the half-life of QM is on the order of seconds, ${ }^{31,32}$ dramatically longer than the former. For comparison, we genetically incorporated the photo-crosslinking Uaa p-azido-phenylalanine (AzF) into GST at position 103, while leaving Ala or Lys at position 107. After photoactivation in cells, both mutants showed GST dimerization (Figure S4), confirming the lack of residue specificity of photocrosslinking via AzF. In addition, the QM-mediated GST(103FnbY/107Lys) has much higher crosslinking efficiency (75\%) than the AzF-mediated GST(103AzF/107Lys) crosslinking (45\%) under the same conditions. These results suggest that longer half-life of QM may enhance crosslinking yield, which will be beneficial for identifying weak and transient interactions.

To enable the use of FnbY in mammalian cells, we tested and optimized its incorporation into green fluorescent protein (GFP) in HeLa cells. The FnbYRS gene was cloned into plasmid pMP-3xtRNA ${ }^{\text {Pyl }}$ and plasmid pNEU-4xtRNA ${ }^{\mathrm{M} 15} .{ }^{33-35}$ The latter uses an optimized tRNA ${ }^{\text {Pyl }}$ to increase Uaa incorporation efficiency in mammalian cells. ${ }^{35}$ We also tested the Y349F mutation in FnbYRS, which has been reported to increase PylRS activity. ${ }^{36}$ The resultant plasmids were separately transfected into the HeLa-GFP-182TAG reporter cells, which contain a genome-integrated GFP gene with a TAG stop codon at site $182 .{ }^{37}$ Suppression of the 182TAG codon would produce full-length GFP rendering cells fluorescent. FACS analysis of transfected cells showed that strong green fluorescence was detected only when cells were fed with FnbY (Figure 4a). In addition, the fluorescence intensity increased with FnbY concentration. Plasmid pNEU-4xtRNA ${ }^{\text {M15-FnbYRS }}$ markedly increased the incorporation efficiency of FnbY in comparison to pMP-3xtRNA ${ }^{\text {Pyl }}$ FnbYRS, and mutation Y349F also enhanced FnbY incorporation (Figure 4a, Figure S5, Figure S6). These data indicate efficient incorporation of FnbY into GFP in HeLa cells.

We then explored photoactivated chemical crosslinking of proteins via FnbY in mammalian

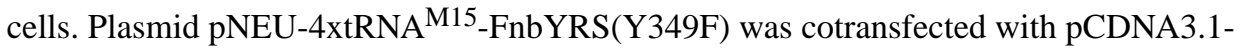
GST(103TAG/107His) into HEK293 cells. Western blot analysis of cell lysate indicate that full-length GST was produced only when FnbY was added to the media (Figure 4b). Consistent with FACS analysis of FnbY incorporation into GFP, both data indicate the high specificity of FnbY incorporation into proteins in mammalian cells. Upon UV illumination of HEK293 cells expressing GST(103TAG/107His), we detected efficient GST dimeric crosslinking through Western blot analysis of cell lysate (Figure 4c). These results 
demonstrate the successful incorporation of FnbY into proteins in mammalian cells and in situ generation of QM for protein crosslinking.

In summary, we genetically encoded FnbY, an unnatural amino acid containing a photocaged QM into proteins in E. coli and mammalian cells. Upon photoactivation FnbY enables the selective bond formation with nine natural amino acid residues in proteins directly in live cells, covering the majority of nucleophilic residues. In comparison with the latent bioreactive Uaas genetically encoded to date, FnbY substantially expands the number of natural residues covalently targetable with a single bioreactive Uaa, and successfully targets Trp, Arg, Met, Gln, and Asn for the first time with proximity-enabled reactivity in vivo. In addition, FnbY's reactivity is photo-controlled, which will promise spatiotemporal resolution for future applications. Also, the strategy of masking and releasing a highly reactive intermediate, represented by QM from FnbY here, may afford a general route to genetically introduce various chemical reactivities inside cells in a biocompatible manner. In comparison with photo-crosslinking Uaas, FnbY reacts with nucleophilic residues selectively, which will enable designer placement of covalent bonds and facilitate detection of crosslinking site by mass spectrometry. The photoactivated intermediate of FnbY, QM, has longer half-life than those of photo-crosslinking Uaas, which can increase crosslinking efficiency and improve the identification of biomolecular interactions. Lastly, QM-bearing small molecules have been versatile in organic synthesis and chemical biology; Genetically encoding FnbY now enables researchers to exploit QM-based chemistries in proteins in live cells. Therefore, we expect that the photoactivatable and multi-targeting reactivity of FnbY will broaden the diversity of proteins and the scope of applications amenable to covalent bonding in vivo for chemical biology, biotherapeutics, and protein engineering.

\section{Supplementary Material}

Refer to Web version on PubMed Central for supplementary material.

\section{ACKNOWLEDGMENT}

L.W. acknowledges the support of the NIH (R01GM118384).

\section{REFERENCES}

(1). Xiang Z; Ren H; Hu YS; Coin I; Wei J; Cang H; Wang L Adding an Unnatural Covalent Bond to Proteins Through Proximity-Enhanced Bioreactivity. Nat. Methods 2013, 10 (9), 885-888. [PubMed: 23913257]

(2). Wang L Genetically Encoding New Bioreactivity. N. Biotechnol 2017, 38 (Pt A), 16-25. [PubMed: 27721014]

(3). Chin JW; Martin AB; King DS; Wang L; Schultz PG Addition of a Photocrosslinking Amino Acid to the Genetic Code of Escherichiacoli. Proc. Natl. Acad. Sci. U. S. A 2002, 99 (17), 1102011024. [PubMed: 12154230]

(4). Hino N; Okazaki Y; Kobayashi T; Hayashi A; Sakamoto K; Yokoyama S Protein Photo-CrossLinking in Mammalian Cells by Site-Specific Incorporation of a Photoreactive Amino Acid. Nat. Methods 2005, 2 (3), 201-206. [PubMed: 15782189]

(5). Zhang M; Lin S; Song X; Liu J; Fu Y; Ge X; Fu X; Chang Z; Chen PR A Genetically Incorporated Crosslinker Reveals Chaperone Cooperation in Acid Resistance. Nat. Chem. Biol 2011, 7 (10), 671-677. [PubMed: 21892184] 
(6). Coin I; Perrin MH; Vale WW; Wang L Photo-Cross-Linkers Incorporated Into G-Protein-Coupled Receptors in Mammalian Cells: a Ligand Comparison. Angew. Chem. Int. Ed. Engl 2011, 50, 8077-8081. [PubMed: 21751313]

(7). Xiang Z; Lacey VK; Ren H; Xu J; Burban DJ; Jennings PA; Wang L Proximity-Enabled Protein Crosslinking Through Genetically Encoding Haloalkane Unnatural Amino Acids. Angew. Chem. Int. Ed. Engl 2014, 53 (8), 2190-2193. [PubMed: 24449339]

(8). Hoppmann C; Lacey VK; Louie GV; Wei J; Noel JP; Wang L Genetically Encoding Photoswitchable Click Amino Acids in Escherichia Coli and Mammalian Cells. Angew. Chem. Int. Ed. Engl 2014, 53 (15), 3932-3936. [PubMed: 24615769]

(9). Hoppmann C; Maslennikov I; Choe S; Wang L In Situ Formation of an Azo Bridge on Proteins Controllable by Visible Light. J. Am. Chem. Soc 2015, 137 (35), 11218-11221. [PubMed: 26301538]

(10). Xuan W; Li J; Luo X; Schultz PG Genetic Incorporation of a Reactive Isothiocyanate Group Into Proteins. Angew. Chem. Int. Ed. Engl 2016, 55 (34), 10065-10068. [PubMed: 27418387]

(11). Coin I; Katritch V; Sun T; Xiang Z; Siu FY; Beyermann M; Stevens RC; Wang L Genetically Encoded Chemical Probes in Cells Reveal the Binding Path of Urocortin-I to CRF Class B GPCR. Cell 2013, 155 (6), 1258-1269. [PubMed: 24290358]

(12). Chen XH; Xiang Z; Hu YS; Lacey VK; Cang H; Wang L Genetically Encoding an Electrophilic Amino Acid for Protein Stapling and Covalent Binding to Native Receptors. ACS Chem. Biol 2014, 9 (9), 1956-1961. [PubMed: 25010185]

(13). Furman JL; Kang M; Choi S; Cao Y; Wold ED; Sun SB; Smider VV; Schultz PG; Kim CH A Genetically Encoded Aza-Michael Acceptor for Covalent Cross-Linking of Protein-Receptor Complexes. J. Am. Chem. Soc 2014, 136 (23), 8411-8417. [PubMed: 24846839]

(14). Kobayashi T; Hoppmann C; Yang B; Wang L Using Protein-Confined Proximity to Determine Chemical Reactivity. J. Am. Chem. Soc 2016, 138 (45), 14832-14835. [PubMed: 27797495]

(15). Hoppmann C; Wang L Proximity-Enabled Bioreactivity to Generate Covalent Peptide Inhibitors of P53-Mdm4. Chemical communications 2016, 52 (29), 5140-5143. [PubMed: 26996321]

(16). Yang B; Tang S; Ma C; Li ST; Shao GC; Dang B; DeGrado WF; Dong MQ; Wang PG; Ding S; Wang L Spontaneous and Specific Chemical Cross-Linking in Live Cells to Capture and Identify Protein Interactions. Nat. Commun 2017, 8 (1), 2240. [PubMed: 29269770]

(17). Tian Y; Jacinto MP; Zeng Y; Yu Z; Qu J; Liu WR; Lin Q Genetically Encoded 2-Aryl-5Carboxytetrazoles for Site-Selective Protein Photo-Cross-Linking. J. Am. Chem. Soc 2017, 139 (17), 6078-6081. [PubMed: 28422494]

(18). Xuan W; Shao S; Schultz PG Protein Crosslinking by Genetically Encoded Noncanonical Amino Acids with Reactive Aryl Carbamate Side Chains. Angew. Chem. Int. Ed. Engl 2017, 56 (18), 5096-5100. [PubMed: 28371162]

(19). Wang N; Yang B; Fu C; Zhu H; Zheng F; Kobayashi T; Liu J; Li S; Ma C; Wang PG; Wang Q; Wang L Genetically Encoding Fluorosulfate-L-Tyrosine to React with Lysine, Histidine, and Tyrosine via SuFEx in Proteins in Vivo. J. Am. Chem. Soc 2018, 140 (15), 4995-4999. [PubMed: 29601199]

(20). Turner AB Quinone Methides. Quarterly Reviews, Chemical Society 1964, 18 (4), 347-360.

(21). Thompson DC; Thompson JA; Sugumaran M; Moldéus P Biological and Toxicological Consequences of Quinone Methide Formation. Chem. Biol. Interact 1993, 86 (2), 129-162. [PubMed: 8448810]

(22). Betley JR; Cesaro-Tadic S; Mekhalfia A; Rickard JH; Denham H; Partridge LJ; Pluckthun A; Blackburn GM Direct Screening for Phosphatase Activity by Turnover-Based Capture of Protein Catalysts. Angew. Chem. Int. Ed 2002, 41 (5), 775-777.

(23). Jiang J; Zeng D; Li S Photogenerated Quinone Methides as Protein Affinity Labeling Reagents. ChemBioChem 2009, 10 (4), 635-638. [PubMed: 19197921]

(24). Toteva MM; Richard JP The Generation and Reactions of Quinone Methides. Adv Phys Org Chem 2011, 45, 39-91. [PubMed: 24511169]

(25). Bai W-J; David JG; Feng Z-G; Weaver MG; Wu K-L; Pettus TRR The Domestication of OrthoQuinone Methides. Acc. Chem. Res 2014, 47 (12), 3655-3664. [PubMed: 25469551] 
(26). Parra A; Tortosa M Para-Quinone Methide: a New Player in Asymmetric Catalysis. ChemCatChem 2015, 7 (10), 1524-1526.

(27). Whidbey C; Sadler NC; Nair RN; Volk RF; DeLeon AJ; Bramer LM; Fansler SJ; Hansen JR; Shukla AK; Jansson JK; Thrall BD; Wright AT A Probe-Enabled Approach for the Selective Isolation and Characterization of Functionally Active Subpopulations in the Gut Microbiome. J. Am. Chem. Soc 2018, 141 (1), 42-47. [PubMed: 30541282]

(28). Nishida M; Harada S; Noguchi S; Satow Y; Inoue H; Takahashi K Three-Dimensional Structure of Escherichia Coli Glutathione S-Transferase Complexed with Glutathione Sulfonate: Catalytic Roles of Cys10 and His106. J. Mol. Biol 1998, 281 (1), 135-147. [PubMed: 9680481]

(29). Dorman G; Prestwich GD Benzophenone Photophores in Biochemistry. Biochemistry 1994, 33 (19), 5661-5673. [PubMed: 8180191]

(30). Knowles JR Photogenerated Reagents for Biological Receptor-Site Labeling. Acc. Chem. Res 2002, 5 (4), 155-160.

(31). Chiang Y; Kresge AJ; Zhu Y Flash Photolytic Generation and Study of P-Quinone Methide in Aqueous Solution. an Estimate of Rate and Equilibrium Constants for Heterolysis of the CarbonBromine Bond in P-Hydroxybenzyl Bromide. J. Am. Chem. Soc 2002, 124 (22), 6349-6356. [PubMed: 12033864]

(32). Zhou QB; Turnbull KD Quinone Methide Phosphodiester Alkylations Under Aqueous Conditions. J. Org. Chem 2001, 66 (21), 7072-7077. [PubMed: 11597232]

(33). Takimoto JK; Dellas N; Noel JP; Wang L Stereochemical Basis for Engineered Pyrrolysyl-tRNA Synthetase and the Efficient in Vivo Incorporation of Structurally Divergent Non-Native Amino Acids. ACS Chem. Biol 2011, 6 (7), 733-743. [PubMed: 21545173]

(34). Fu C; Kobayashi T; Wang N; Hoppmann C; Yang B; Irannejad R; Wang L Genetically Encoding Quinoline Reverses Chromophore Charge and Enables Fluorescent Protein Brightening in Acidic Vesicles. J. Am. Chem. Soc 2018, 140 (35), 11058-11066. [PubMed: 30132658]

(35). Serfling R; Lorenz C; Etzel M; Schicht G; Böttke T; Mörl M; Coin I Designer tRNAs for Efficient Incorporation of Non-Canonical Amino Acids by the Pyrrolysine System in Mammalian Cells. Nucleic Acids Res 2018, 46 (1), 1-10. [PubMed: 29177436]

(36). Yanagisawa T; Ishii R; Fukunaga R; Kobayashi T; Sakamoto K; Yokoyama S Multistep Engineering of Pyrrolysyl-tRNA Synthetase to Genetically Encode N(Epsilon)-(OAzidobenzyloxycarbonyl) Lysine for Site-Specific Protein Modification. Chem. Biol 2008, 15 (11), 1187-1197. [PubMed: 19022179]

(37). Wang W; Takimoto JK; Louie GV; Baiga TJ; Noel JP; Lee K-F; Slesinger PA; Wang L Genetically Encoding Unnatural Amino Acids for Cellular and Neuronal Studies. Nat. Neurosci 2007, 10 (8), 1063-1072. [PubMed: 17603477] 
a<smiles>NC(C(=O)O)C(F)c1ccc(OCc2ccccc2[N+](=O)[O-])cc1</smiles>
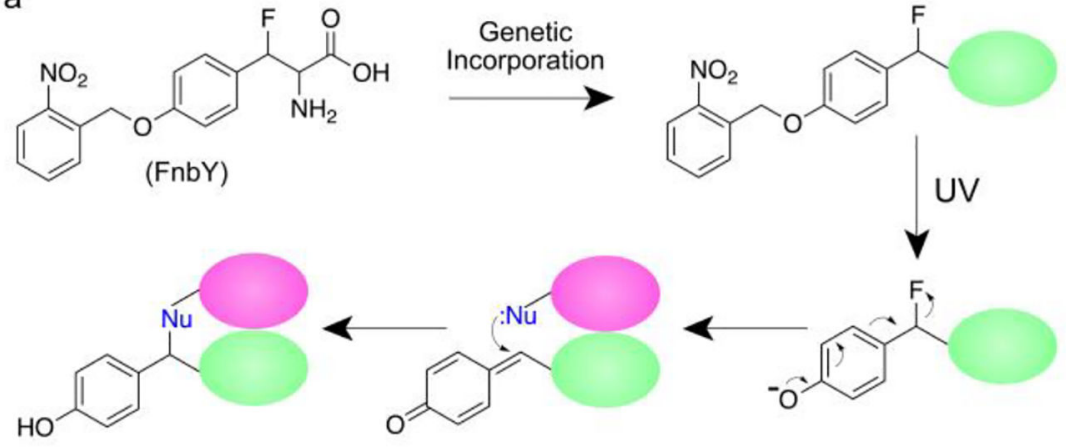

b
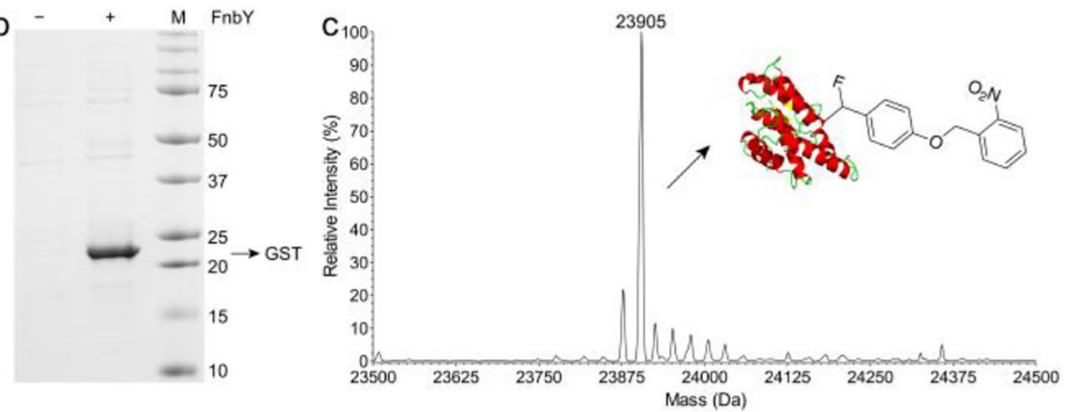

Figure 1.

Genetically encode FnbY into GST protein in E. coli. (a) Scheme showing FnbY structure and photoactivated Michael addition. Photoactivation of FnbY generates QM on protein in situ, which reacts with nucleophilic residues in proximity via Michael addition, forming new covalent bonds. (b) SDS-PAGE analysis of FnbY incorporation into GST at site 103. (c) ESI-TOF MS analysis of purified GST(103FnbY). Expected: 23906 Da; measured: 23905 Da. 
a

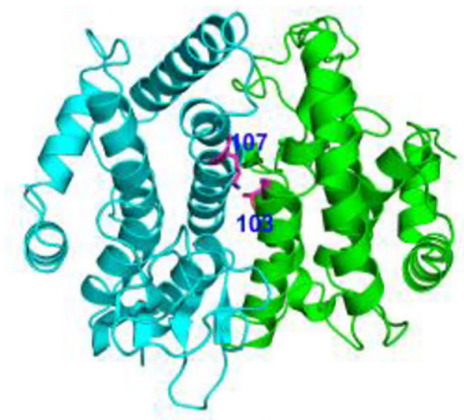

C

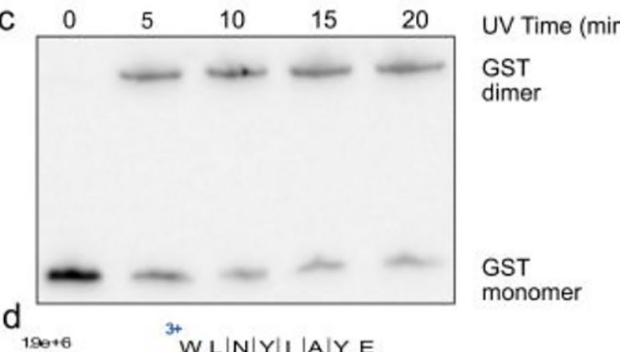

b
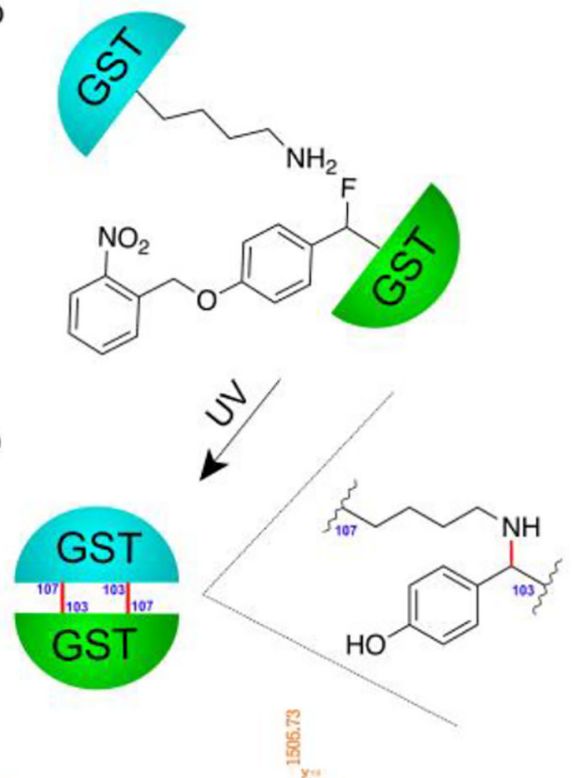

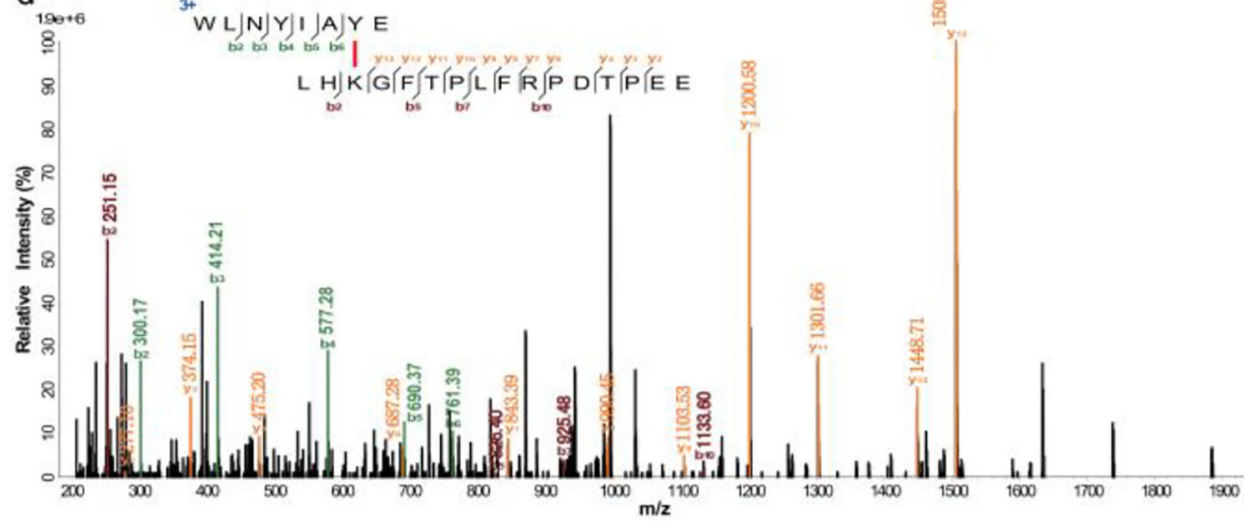

Figure 2.

Photoactivated FnbY selectively reacts with a proximal Lys in protein in E. coli cells. (a) Crystal structure of E. coli GST (PDB code 1A0F) showing site 103 and 107 at the dimer interface. (b) Scheme showing photoactivated chemical crosslinking via FnbY: FnbY103 incorporated in GST, upon photoactivation, reacts with the nearby Lys107 of the other GST monomer, resulting in a covalently crosslinked GST dimer. (c) GST dimerization detected on Western blot of cell lysates of E. coli cells expressing GST(103FnbY). Cells were illuminated with UV light at $302 \mathrm{~nm}$ for indicated duration. (d) Tandem mass spectrum of GST(103FnbY) after photoactivated chemical crosslinking. A series of $b$ and y ions indicate the crosslinking of FnbY103 (in final form of Tyr) with Lys107. 

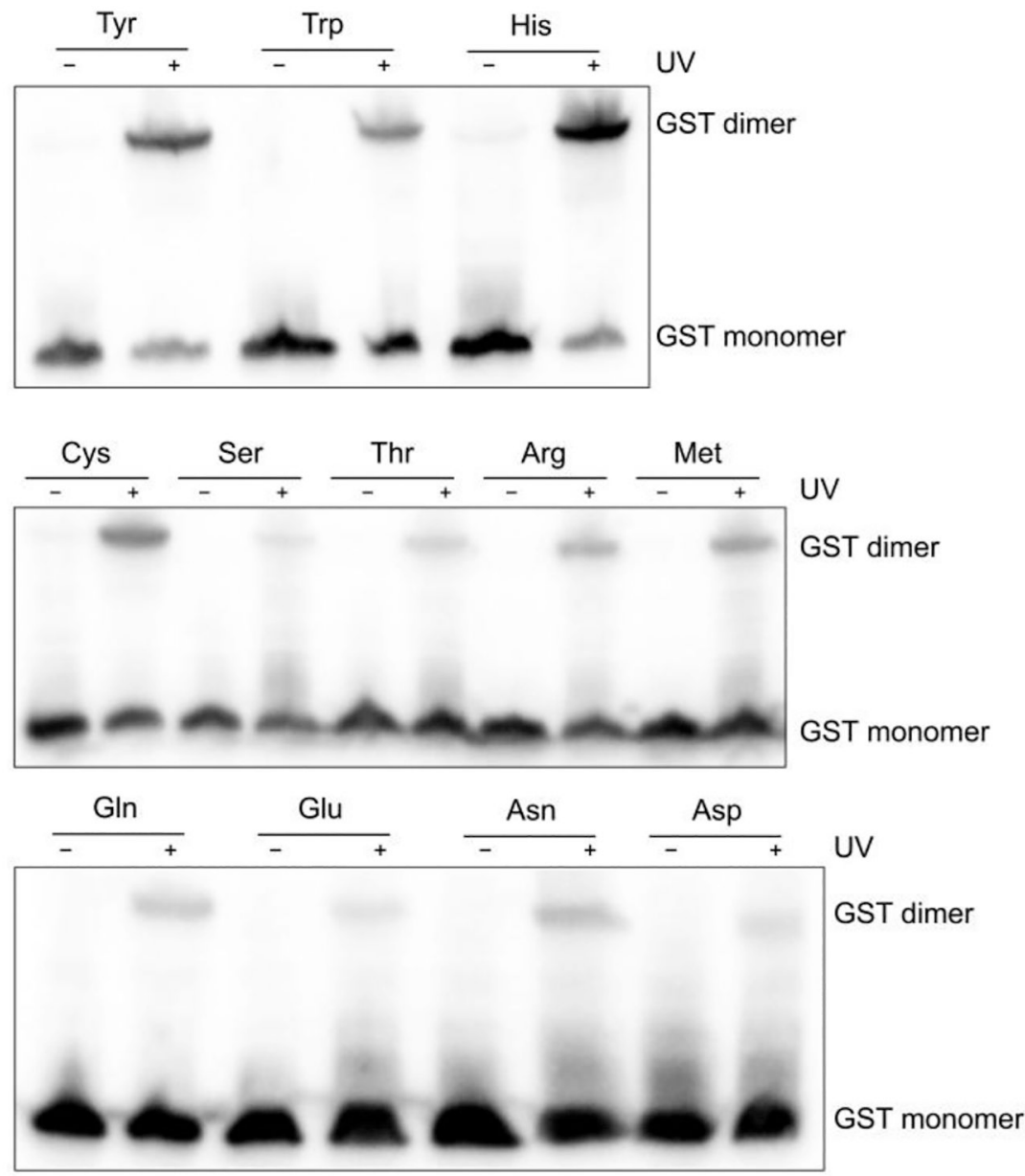

Figure 3.

Photoactivated FnbY reacts with multiple residues in proteins in E. coli. E. coli cells expressing GST mutants with FnbY103 and position 107 mutated to the indicated residues were illuminated with UV light, and cell lysates were analyzed with Western blot to detect GST. 

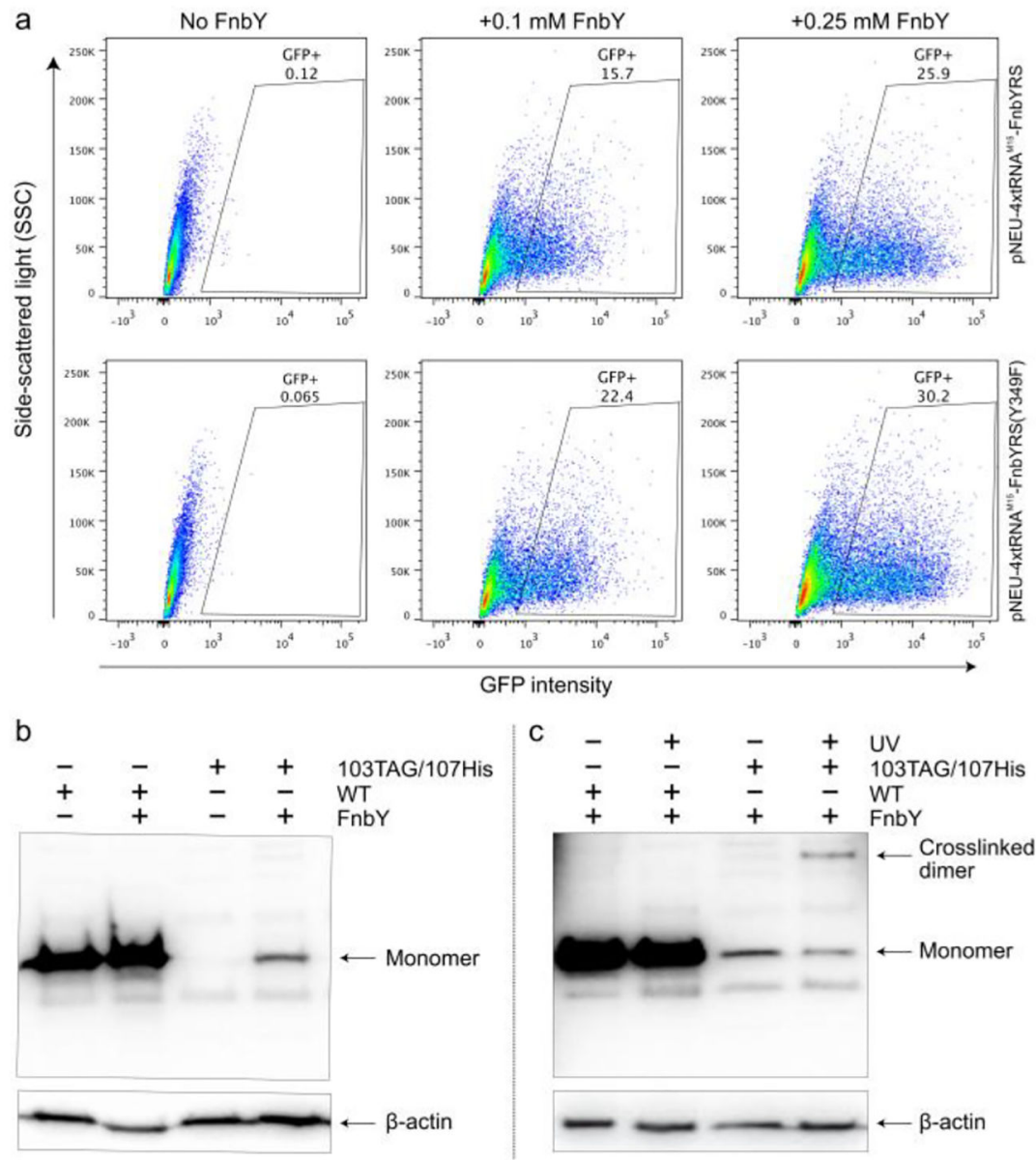

Figure 4.

Genetic incorporation of FnbY into proteins in mammalian cells and in cell photoactivated chemical crosslinking of GST. (a) FACS analysis of FnbY incorporation into GFP in HeLa cells. HeLa-GFP-182TAG stable cell line was transfected with pNEU-4xtRNA ${ }^{\text {M15 }}$-FnbYRS or pNEU-4xtRNA ${ }^{\mathrm{M} 15}$-FnbYRS(Y349F) and cultured with indicated concentrations of FnbY. (b) Western blot analysis of incorporation of FnbY into GST(103TAG/107His) in HEK293 cells. (c) Western blot analysis of photoactivated chemical crosslinking of GST(103FnbY/ 107His) in HEK293 cells. 